\title{
BMJ Open Value of lung ultrasound for the diagnosis of COVID-19 pneumonia: a protocol for a systematic review and meta-analysis
}

\author{
Yujiao Yang (D) , ${ }^{1,2}$ Donghang Zhang, ${ }^{1}$ Cheng Zhou, ${ }^{3}$ Han Huang, ${ }^{4}$ Rurong Wang ${ }^{1}$
}

To cite: Yang Y, Zhang D, Zhou $\mathrm{C}$, et al. Value of lung ultrasound for the diagnosis of COVID-19 pneumonia: a protocol for a systematic review and meta-analysis. BMJ Open 2020;10:e039180. doi:10.1136/ bmjopen-2020-039180

- Prepublication history for this paper is available online. To view these files, please visit the journal online (http://dx.doi. org/10.1136/bmjopen-2020039180).

YY and DZ contributed equally.

Received 08 April 2020

Revised 02 June 2020

Accepted 11 June 2020

\section{Check for updates}

(c) Author(s) (or their employer(s)) 2020. Re-use permitted under CC BY-NC. No commercial re-use. See rights and permissions. Published by BMJ.

${ }^{1}$ Department of Anesthesiology, Sichuan University West China Hospital, Chengdu, China ${ }^{2}$ Department of Anesthesiology, Affiliated Hospital of North Sichuan Medical College, Nanchong, China

${ }^{3}$ Lab of Anesthesia and Critical Care Medicine, Translational Neuroscience Center, Sichuan University West China Hospital, Chengdu, Sichuan, China ${ }^{4}$ Department of Anesthesiology, Sichuan University West China Second University Hospital, Chengdu, Sichuan, China

Correspondence to Professor Rurong Wang; wangrurong@scu.edu.cn

\section{ABSTRACT}

Introduction In the recent COVID-19 pandemic, cases have exceeded over one million, with the number of confirmed cases increasing by $50000-60000$ per day. The virus has killed nearly 50000 people all over the world in only 3 months. These reforms bring major challenges to the public health and healthcare system. The pulmonary pathological features during the initial phase of COVID-19 are alveolar oedema, pneumocyte hyperplasia, gravitational consolidations and interstitial thickening. The ability of lung ultrasound (LUS) and its evolving applications in the diagnosis of COVID-19 pneumonia are widespread. This study aims to evaluate the surveillance value of LUS in the diagnosis of COVID-19 pneumonia. Methods and analysis We will perform a systematic search and meta-analysis on the use of LUS to diagnose and confirm COVID-19 pneumonia. We will search Ovid Medline, Ovid Embase, Web of Science, Cochrane Library, Scopus, Google Scholar, China Biology Medicine disc and WHO Global Health Library for studies on diagnostic accuracy from December 2019 to April 2021. Data collection and screening will be individually accomplished by two reviewers. The assessment of risk of bias for each outcome will be conducted using the QUADAS-2 (Quality Assessment of Diagnostic Accuracy Studies 2) tool. Data will be synthesised and heterogeneity will be evaluated. Meta-analysis will be conducted when strong homogeneous data are accessible. Grading of Recommendations Assessment, Development and Evaluation(GRADE) will be used to assess quality of evidence.

Ethics and dissemination Approval of ethics committee is not needed for this review. While results will be disseminated electronically, effective dissemination will be done through presentations and peer-reviewed publication. PROSPERO registration number CRD42020177803; pre-results.

\section{INTRODUCTION}

\section{Rationale}

In the recent COVID-19 pandemic, cases have exceeded over one million, with the number of confirmed cases increasing by 50000-60000 per day. The virus has killed nearly 50000 people all over the world in only 3 months. These reforms bring major
Strengths and limitations of this study

- Our review will be able to answer important questions and clarify confusions regarding the specificity and sensitivity of lung ultrasound (LUS) in diagnosing COVID-19 pneumonia.

- Studies on the basis of the Preferred Reporting Items for Systematic Review and Meta-Analysis Protocols 2015 will be included to review the diagnosis of COVID-19 pneumonia using LUS.

- We will conduct subgroup analysis according to the level of sonographers.

- The literature will be screened independently by two reviewers: first the title and abstracts will be reviewed and then the text will be screened extensively.

Due to differences in departments, clinicians and countries, there may be heterogeneity in the results.

challenges to the public health and healthcare system. In the absence of effective therapeutic measures and vaccinations for COVID-19, identification of disease patterns during its early stages is of great importance. Reverse transcription (RT)-PCR remains critical in the confirmation of COVID-19 infection; however, a significant number of negative results from throat swab samples have been observed in $40 \%-70 \% .^{1-3}$ In addition to the unavailability of RT-PCR during the early stages of outbreaks, this highlights the need for an easier and faster method to identify COVID-19 cases, especially in countries with limited resources. Chest CT findings can detect the initial phases of the disease, such as ground-glass opacity and consolidation, interstitial changes, and peripheral lung distribution. ${ }^{45}$ However, the radiation, the high cost and the fixed rooms for chest CT make it inconvenient in clinical application.

The pulmonary pathological features during the initial phase of COVID-19 are alveolar oedema, pneumocyte hyperplasia, gravitational consolidations and interstitial 
thickening. ${ }^{6}$ The clinical application of lung ultrasound (LUS) has become sufficiently known and its use widespread $^{7-9}$ This review aims to examine the surveillance value of LUS in detecting COVID-19 pneumonia.

\section{Aims and objectives}

The principal objective of our review is to conduct a diagnostic test accuracy (DTA), meta-analysis and systematic review to determine the specificity and sensitivity of LUS, which will be correlated with the diagnostic results of RT-PCR for COVID-19 pneumonia. Here we hypothesised that LUS is a more specific and sensitive technique for investigating patients with COVID-19 pneumonia. Our secondary objective is to investigate potential sources of heterogeneity among the study population, data source, validation method, and diagnosis and time period.

\section{METHODS AND ANALYSIS}

We will carry out the meta-analysis and systematic review on the basis of the Preferred Reporting Items for Systematic Review and Meta-Analysis Protocols (PRISMA-P), checklist and detailed explanation of scoping reviews, and the Cochrane DTA reviews (Cochrane Handbook). This protocol follows the format recommended by the PRIMA-P guidelines. ${ }^{10}$

\section{Inclusion criteria}

Studies included in this review should satisfy all of the following standards recommended for reviews of DTA.

\section{Type of studies}

We will include original studies incorporating diagnostic tests, with both prospective and retrospective studies being included. Randomised trials can only be included if individuals received both the index test and a reference standard, while case reports will be excluded. There will be no limitations on language and publication status.

\section{Participants}

We will include studies enrolling patients of all ages who have a positive PCR for COVID-19 pneumonia and of any gender in clinical settings.

\section{Index tests}

Studies that use LUS to test for COVID-19 pneumonia will be included. We excluded studies with non-human studies and studies with data that cannot be extracted, as well as non-original studies (reviews, author responses, comments) or secondary research (systematic review, meta-analysis).

\section{Comparison}

Evaluation of the diagnostic accuracy of LUS was performed according to the gold standard for diagnosis of COVID-19 pneumonia (LUS should not be part of the gold standard). Currently RT-PCR is the gold standard for detection of COVID-19 pneumonia.

\section{Outcomes}

Studies that will be included in this review must report at a minimum one of the following estimated validations: specificity, sensitivity, negative predictive value and positive predictive value, or if not reported the data have to be retrievable by calculation.

\section{Information sources}

We will search Ovid Medline, Ovid Embase, Web of Science, Cochrane Library, Scopus, Google Scholar, WHO Global Health Library and China Biology Medicine disc for studies on diagnostic accuracy from December 2019 to April 2021. There will be no restrictions to language and place.

To check further published trials, we will be in close contact with the study authors of the included trials to identify additional trials, whether published or unpublished.

Last, we will manually search for objective studies through reference lists having retrieved citations, related grey literature and prior reviews on the topic.

\section{Search strategy}

The search strategy will comprise subject heading terms and free text (title and abstract) on COVID-19 pneumonia.

We will also review the list of citations and systematic reviews associated with the study to investigate effective studies. Table 1 shows the search process for PubMed. The search procedure will be revised according to other databases.

\section{Study selection}

The results of the literature search will be imported into EndNote V.X9. Two authors (DZ and YY) will review the title and abstract independently. There will be no restrictions to the protocol of LUS used to diagnose COVID-19 pneumonia and no restrictions to the type of physician and type of machine or transducer. The entire text of the journal articles that meet the eligibility criteria of this review will be acquired and will be reviewed by two authors (DZ and $\mathrm{YY}$ ). During the entire text screening, the reasons for rejection of articles will be marked with respect to a categorised list: (1) duplicate study, (2) did not conduct validation, (3) did not obtain full text, (4) wrong index, (5) not a suitable comparator, or (6) no estimates for validation or inadequate data for calculation. Dissimilarities will be solved by discussion with a third author (RW) when needed.

\section{Collection of data}

Two investigators (YY and DZ) will independently use the same predesigned forms to collect the primary summary outcome and modifiers in each study, and dissimilarities between the two evaluators will be resolved on consultation with a third author (RW). In every study, the following data will be extracted: author, journal, publication time, population size, study setting, mean age, index test details (LUS) and reference test (RT-PCR), sonographer, 


\begin{tabular}{|c|c|}
\hline Number & Search terms \\
\hline \#1 & Ultrasonography (Mesh terms) \\
\hline \#2 & Ultrasonography (title/abstract) \\
\hline \#3 & Ultrasonic (title/abstract) \\
\hline \#4 & diagnostic imaging (title/abstract) \\
\hline \#5 & ultrasound (title/abstract) \\
\hline \#6 & Echography (title/abstract) \\
\hline \#7 & Ultrasound* (title/abstract) \\
\hline \#8 & Ultrasonic (title/abstract) \\
\hline \#9 & Echotomography (title/abstract) \\
\hline \#10 & $\begin{array}{l}\text { \#1 OR \#2 OR \#3 OR \#4 OR \#5 OR \#6 OR \#7 } \\
\text { OR\#8 OR\#9 }\end{array}$ \\
\hline$\# 11$ & 2019 nCoV (title/abstract) \\
\hline \#12 & 2019 novel coronavirus (title/abstract) \\
\hline \#13 & COVID 19 (title/abstract) \\
\hline$\# 14$ & COVID19 (title/abstract) \\
\hline \#15 & new coronavirus (title/abstract) \\
\hline \#16 & novel coronavirus (title/abstract) \\
\hline$\# 17$ & SARS CoV-2 (Title/Abstract) \\
\hline \#18 & SARS-CoV (title/abstract) \\
\hline \#19 & 2019-nCoV (title/abstract) \\
\hline \#20 & $\begin{array}{l}\# 11 \text { OR \#12 OR \#13 OR \#14 OR \#15 OR \#15 OR } \\
\# 17 \text { OR\#18 OR \#19 }\end{array}$ \\
\hline \#21 & \#10 AND \#20 \\
\hline
\end{tabular}

recruitment methods, true positives, true negatives, false positives, false negatives, specificity, sensitivity, bias assessment by study authors, bias assessment by review authors and source of funding.

\section{Subgroup analysis}

After obtaining permission to use data, we will perform a subgroup analysis on various levels of sonographers to evaluate whether diagnostic accuracy varies in different subgroups. We are aware that an ultrasound examination is an operator-dependent technique and that it is associated with a learning curve. Different studies may have different skilled sonographers.

\section{Risk of bias assessment}

Two independent reviewers (DZ and $\mathrm{YY}$ ) will use the QUADAS-2 (Quality Assessment of Diagnostic Accuracy Studies 2) tool to assess bias and evaluate studies on diagnostic accuracy. ${ }^{11}$ Included studies will meet the under conditions: patient selection, index test, reference standard, flow and timing. A pilot programme for quality evaluation will be used in three studies in duplicate in order to clarify the criteria for assessment and optimise reproducibility. Review Manager V.5 (http://ims.cochrane. org/revman/download) will be used for this process. Each dissimilarity will be solved by suggestions from the senior author (RW).

\section{Strategy for data synthesis}

The initial steps of data synthesis will involve calculation of statistics including specificity and sensitivity with corresponding 95\% CI and positive and negative likelihood ratios from diagnostic $2 \times 2$ tables of primary studies. Descriptive data analysis will be illustrated using forest plot and summary receiver operating characteristic curve. Data on LUS for diagnosis of COVID-19 pneumonia will be gathered from primary accuracy studies. In addition, the research group will evaluate the studies for clinical and methodological diversity by comparing significant characteristics such as studies related to patients, LUS acquisition, design and interpretation. The $\mathrm{I}^{2}$ statistics and $Q$ test will be used to measure and interpret statistical diversity, ${ }^{12}$ after which the results will be concluded.

An $\mathrm{I}^{2}$ value more than $75 \%$ indicates significant heterogeneity and if encountered the data will not be combined. A bivariate random-effects regression method will be applied to estimate specificity and sensitivity.

We will further evaluate meta-regression and subgroup analyses using some modifiers. Funnel curve will be plotted for publication bias. Complete data analyses will be evaluated using Stata (Version 12.0; Stata Corporation). and RevMan (http://ims.cochrane.org/revman/ download).

\section{DISCUSSION}

LUS has advantages in terms of bedside evaluation, safety, convenience and the probability of repeat examination. ${ }^{13}$ More importantly, the probability of bedside evaluation with LUS decreases the need for patient transfers and minimises potential transmission among healthcare workers during this special time. Real-time monitoring by LUS may designing a proper diagnostic workup according to the general and local technological and human resources when compared with RT-PCR for diagnosing COVID-19 pneumonia.

As a matter of fact, LUS has not been considered an alternative in the guidelines for imaging diagnosis of COVID-19 pneumonia. Therefore, more clinical evidence is needed to support it. We think clinical outcomes are likely achieved when LUS is used to diagnose COVID-19 pneumonia. The histopathology during the initial phases of COVID-19 pneumonia is characterised by diffuse alveolar damage with vascular congestion, patchy inflammatory cellular infiltration (B lines) and intra-alveolar oedema, while interstitial thickening and patchy reparative processes show ongoing reparative process. The progressive stage of COVID-19 pneumonia manifests as gravitational consolidation, which is similar to acute respiratory distress syndrome. Meanwhile, alveolar congestion, haemorrhagic necrosis, flaking, oedema and fibrosis may exist (white lung). Therefore, pulmonary consolidation can be graded using LUS images of patients with viral infection. Topographic images of the lesions can also be developed under ultrasound. Furthermore, the extent of damage of the lesions on the lung surface and their 
progression or regression over time can also be examined using LUS. In fact, many studies have reported the use of LUS in detecting COVID-19 pneumonia. The goal of the present study is to provide a procedure for meta-analysis and systematic review to review LUS for COVID-19 pneumonia. In this study we explore the accuracy of LUS in diagnosing COVID-19 pneumonia. The results of this study will provide clinical evidence on the diagnostic validity of LUS in identifying COVID-19. We hope to provide effective information for public health policymakers and patients regarding the use of LUS for diagnosis and treatment of COVID-19 pneumonia.

\section{ETHICS AND DISSEMINATION}

This review and meta-analysis involves secondary data and does not require ethics approval. The outcomes of this study will be presented internationally. These results will be reported in a peer-reviewed scientific journal. The full review will be distributed in printed form, electronically and on appropriate social media sites.

\section{Twitter Yujiao Yang @Jillyan36779495}

Contributors YY and DZ conceived the idea of this study, and will screen the titles, abstracts and full text to identify studies for inclusion or exclusion, extract data and input data, and assess risk of bias. $\mathrm{CZ}$ and $\mathrm{HH}$ provided statistical advice and revision of the final text. RW provided feedback on disagreements between the two authors and reviewed the protocol.

Funding This research work received contributions from the 1.3 .5 project of disciplines excellence development-Clinical Research Incubation Project, West China Hospital of Sichuan University (2018HXFH029) and Project of North Sichuan Medical College (CBY18-A-YB47).

Disclaimer The funder did not contribute to protocol development. Competing interests None declared.

Patient and public involvement Patients and/or the public were not involved in the design, or conduct, or reporting, or dissemination plans of this research.

Patient consent for publication Not required.

Provenance and peer review Not commissioned; externally peer reviewed.

Open access This is an open access article distributed in accordance with the Creative Commons Attribution Non Commercial (CC BY-NC 4.0) license, which permits others to distribute, remix, adapt, build upon this work non-commercially, and license their derivative works on different terms, provided the original work is properly cited, appropriate credit is given, any changes made indicated, and the use is non-commercial. See: http://creativecommons.org/licenses/by-nc/4.0/.

ORCID iD

Yujiao Yang http://orcid.org/0000-0002-5409-6007

\section{REFERENCES}

1 Wu Z, McGoogan JM. Characteristics of and important lessons from the coronavirus disease 2019 (COVID-19) outbreak in China: summary of a report of 72314 cases from the Chinese center for disease control and prevention. JAMA2020.

$2 \mathrm{Ai}$ T, Yang Z, Hou H, et al. Correlation of chest CT and RT-PCR testing for coronavirus disease 2019 (COVID-19) in China: a report of 1014 cases. Radiology 2020;296:E32-40.

3 Fang Y, Zhang H, Xie J, et al. Sensitivity of chest CT for COVID-19: comparison to RT-PCR. Radiology 2020;296:E115-7.

$4 \mathrm{Li} \mathrm{K}, \mathrm{Wu}$ J, Wu F, et al. The clinical and chest CT features associated with severe and critical COVID-19 pneumonia. Invest Radiol 2020;55:327-31.

5 Li Y, Xia L. Coronavirus disease 2019 (COVID-19): role of chest CT in diagnosis and management. AJR Am J Roentgenol 2020;214:1280-6.

6 Soldati G, Smargiassi A, Inchingolo R, et al. Is there a role for lung ultrasound during the COVID-19 pandemic? J Ultrasound Med 2020;39:1459-62.

7 Yousef N, De Luca D. The role of lung ultrasound in viral lower respiratory tract infections. Am J Perinatol 2018;35:527-9.

8 Berce V, Tomazin M, Gorenjak M, et al. The usefulness of lung ultrasound for the aetiological diagnosis of community-acquired pneumonia in children. Sci Rep 2019;9:17957.

9 Lissaman C, Kanjanauptom P, Ong C, et al. Prospective observational study of point-of-care ultrasound for diagnosing pneumonia. Arch Dis Child 2019;104:12-18.

10 Macaskill PGC, Deeks JJ, Harbord RM, et al. Chapter 10: Analysing and Presenting Results. In: Deeks JJ, Bossuyt PM, Gatsonis C, et al, eds. Cochrane handbook for systematic reviews of diagnostic test accuracy version 1.0. The Cochrane Collaboration, 2010. http:// srdtacochraneorg/

11 Deeks JJ WS, Davenport C. Chapter 4: Guide to the contents of a Cochrane Diagnostic Test Accuracy Protocol. In: Deeks JJ, Bossuyt PM, Gatsonis C, eds. Cochrane handbook for systematic reviews of diagnostic test accuracy version 1.0.0. The Cochrane Collaboration, 2013. http://srdta.cochrane.org/. 2013

12 Higgins JPT, Thompson SG. Quantifying heterogeneity in a metaanalysis. Stat Med 2002;21:1539-58.

13 Pereda MA, Chavez MA, Hooper-Miele CC, et al. Lung ultrasound for the diagnosis of pneumonia in children: a meta-analysis. Pediatrics 2015;135:714-22. 